\title{
Visual Prosthesis : Artificial Vision
}

\author{
Brig A Banarji*, Col VS Gurunadh ${ }^{+}$, Col S Patyal ${ }^{\#}$, Col TS Ahluwalia**, Maj Gen DP Vats, sm, vsm ${ }^{++}$, \\ Col M Bhadauria (Retd) \#\#
}

MJAFI 2009; 65 : 348-352

Key Words : Artificial vision; Prosthesis

\begin{abstract}
"The next care to be taken, in respect of the Senses, is a supplying of their infirmities with instruments, and as it were, the adding of artificial organs to the natural... and as glasses have promoted our seeing, so 'tis not improbable, but that there may be found many mechanical inventions to improve our other senses of hearing, smelling, tasting and touching. "
\end{abstract}

Robert Hooke English natural philosopher (1665) [1]

Nothing could demonstrate more than this concept of Hooke with regards to replacement of all the senses by artificial means. Hooke was enthralled by spectacle correction itself and felt that the other senses could also be improved. No doubt the medical world has caught up with hearing aids and cochlear implants but in the real sense the restoration of sight to a blind is still in an experimental stage and the most daunting challenge in ophthalmic research.

\section{Introduction}

$\mathrm{I}_{\mathrm{te}}^{\mathrm{n}}$ the normal visual pathway, light travels through the tear film, cornea, aqueous, pupil, lens, and vitreous, to activate the light sensitive photoreceptors and set up the trans-synaptic connections of the retina (Fig.1). In cases where there is gross destruction of the eye like phthisis bulbi, restoration of vision is not possible. But in conditions where blindness is due to a disease in the photoreceptors in the retina like Retinitis Pigmentosa (RP) (Fig. 2) or Age Related Macular Degeneration (AMD) (Fig.3 a,b) wherein the neural connections are intact, in cortical tumours and lesions involving the visual pathways or occipital cortex wherein the photoreceptors are intact, there exists a scientific possibility wherein a device can be implanted at any location in this pathway to set up a neuronal electrical stimulation. Such a device is known as a visual prosthesis or bionic eye and the vision created is known as artificial vision. Incidentally, RP is the leading inherited cause of blindness, with 1.5 million people affected worldwide [2]. AMD seen in adults over 65 years is the leading cause of visual loss with 700,000 new cases diagnosed annually in the USA and $10 \%$ of whom become legally blind each year. Moreover, these are a group of blind people who have had good vision all their life and have become blind at a time when all faculties are on the decline and daily survival requires vision [3].

Depending upon location along the visual pathway this prosthetic device could be in the visual cortex, on the optic nerve or at the retina.

\section{History}

The history of visual prosthesis dates back to the understanding of electrical stimulation of human tissues in 1799. It was Foerster a German neurosurgeon who in 1929 demonstrated that electrical stimulation of the visual cortex could lead to the perception of light spot (phosphene) by the subject. The first prototype of a device was conceived by Brindley in 1960s who improvised a device on the cerebral cortex.

\section{Cortical Prosthesis}

Working on the concepts of Foerster, Giles Brindley along with Dobelle pioneered all subsequent work in the development of a visual prosthesis. They demonstrated that phosphenes and patterned perceptions could be evoked, by electrically stimulating the occipital cortex by implanted electrodes [3]. Both researchers implanted arrays with over 50 electrodes subdurally over the occipital pole, thus providing evidence of the ability to return the sensation of vision to individuals who had severed visual pathway anterior to the visual cortex. Dobelle's 64 channel platinum electrode surface stimulation prosthesis allowed blind patients to recognise six inches characters at a distance of five feet [4].

Since most of the visual cortex lies deep within the calcarine fissure and is inaccessible to cortical surface electrodes, intracortical stimulation was introduced in

"Professor \& Head, ${ }^{*}$ Reader, ${ }^{*}$ Associate Professor (Ophthalmology) AFMC, Pune-40. ${ }^{* *}$ Senior Advisor (Ophthalmology), Command Hospital (NC). ${ }^{++}$MG (Med) HQ Southern Command, Pune. ${ }^{\#}$ CMO, Sitapur Eye Hospital, Sitapur, UP.

Received : 21.01.09; Accepted : 07.07.09

E-mail : ajaybanarji@rediffmail.com 
the hope of remedying the shortcomings of surface stimulation via low current high-fidelity systems.

Current models of the intracortical prosthesis include the Utah electrode array [3] and the Illinois Intracortical Visual Prosthesis project [5]. The Utah device consists of multiple silicon spikes organised in a square grid measuring $4.2 \times 4.2 \mathrm{~mm}$ with a platinum electrode at the tip of each spike [3]. The Ilinois device consisting of 152 intracortical microelectrodes, has been chronically implanted in an animal model and the model for human implantation has been proposed [5].

The cortical visual prosthesis is advantageous over other approaches as it bypasses all diseased visual pathway neurons rostral to the primary visual cortex. However, the disadvantages are the histological changes induced by a chronically implanted cortical prosthesis which needs to be investigated. The organisation of the visual field is very complex at the level of the primary cortex than at the retina or optic nerve. It is not easily reproducible between various patients and there is a high level of cortical specialisation of various parameters including colour, motion, and eye movement [6]. Finally, surgical complications of this approach carry significant morbidity and mortality for the patient.

\section{Optic Nerve Prosthesis}

Investigators have targeted the optic nerve as a potential site for the implantation of a visual prosthesis. Veraart et al [7], have attempted this method by employing the concept of a spiral nerve cuff electrode. An electrode cuff is surgically implanted circumferentially on the external surface of the optic nerve. Since the device does not penetrate the optic nerve sheath, it relies on the principle of retinotopic organisation within the optic nerve. Recently, optic nerve prosthesis with four electrodes has been implanted into a human patient.

The optic nerve is an appealing site for the implementation of a visual prosthesis as the entire visual field is represented in a small area which can be reached surgically and presents a viable anatomic location for an implant. However, the optic nerve is a densely consolidated neural structure with approximately 1.2 million axons in a $2 \mathrm{~mm}$ diameter cylinder. Surgical manipulation of this area requires dissection of the dura, with an inherent risk of infections and possibly interruption of blood flow to the optic nerve [3]. The optic nerve and the retinal ganglion cells (RGC) represent higher- order structures than the bipolar cells targeted by the retinal prosthesis. As such the processing power of the bipolar, amacrine and horizontal cells is lost and therefore much more image processing must be achieved by the implant rather than relying on intact human physiologic pathways. Lastly, the nerve fibres from the macula lie most centrally within the optic nerve. The cuff electrodes are thus, further away from the macular fibres and this will dramatically limit the use of this approach. Investigators have proposed intraneuronal stimulation devices in order to target individual neurons within the optic nerve more accurately [3].

Recently Veraart et al [8] published results of an optic nerve prosthesis chronically implanted in a blind volunteer of RP with no residual vision. The volunteer underwent performance evaluation during the course of a training programme. The results were encouraging in that the blind volunteer was able to interact with the environment adequately while demonstrating pattern recognition and a learning effect for processing time and orientation discrimination.

\section{Retinal Prosthesis}

The basis for the retinal prosthesis is the post-mortem morphometric analysis of the retina of patients with endstage RP which has revealed that $78.4 \%$ of inner nuclear and $29.7 \%$ of ganglion layer cells were retained compared to only $4.9 \%$ of photoreceptors [3]. It was also seen in legally blind neovascular AMD patients that $93 \%$ of RGCs were spared and an increase in inner nuclear layer cells was noted by $10 \%[9,10]$. These results imply that theoretically it is possible to stimulate the remaining retinal neurons electrically to elicit useful visual perceptions. These initial observations have led to the development of intraocular retinal prostheses in various centres. These retinal prostheses could be epiretinal when the device is implanted into the vitreous cavity and attached to the inner retinal surface, so the electrical stimulation meets the inner retina first. In the second approach the device is implanted in the potential space between the retinal pigment epithelium and the neurosensory retina, so the electrical stimulation meets the outer retina first.

\section{Epiretinal Prosthesis}

Epiretinal implants rely on imaging devices such as a camera and then transform this visual information to patterns of electrical stimulation to excite remaining viable retinal neurons.

Humayun et al [3], have pioneered research in the development of a device called the intraocular retinal prosthesis (IRP). This IRP includes both an external, wearable component and an implantable, intraocular component. The external component consists of a spectacle incorporated with a light weight camera, pocket batteries and a visual processing unit. Power and data is sent by wireless link from the external unit to the internal portion of the prosthesis. The intraocular portion consists of a receiver, stimulating chip and a microelectrode array including 16 platinum electrodes 
of approximately 500 um in diameter. The electrode array is affixed to the epiretinal surface by a retinal tack. This model captures digital video and translates it to a pixelated image externally. The final processed data and power is then transferred to the implanted portion of the prosthesis. The implanted portion of the device then converts the transferred visual data into controlled patterns of electrical pulses to stimulate the remaining retinal neurons. In preliminary tests in the operating room with temporary epiretinal devices [3] patients could identify crude forms such as letters or a box shape.

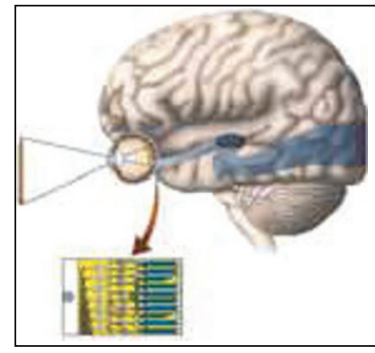

Fig. 1 : Human visual system [3]
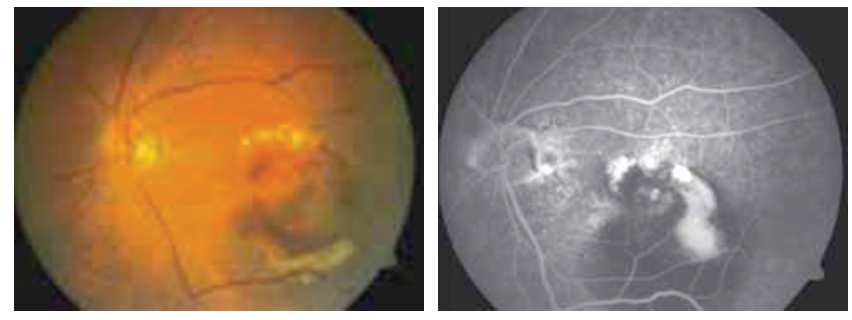

Fig. 3 : (a) Age-related macular degeneration (AMD) and (b) corresponding fundus fluorescein angiogram of the case

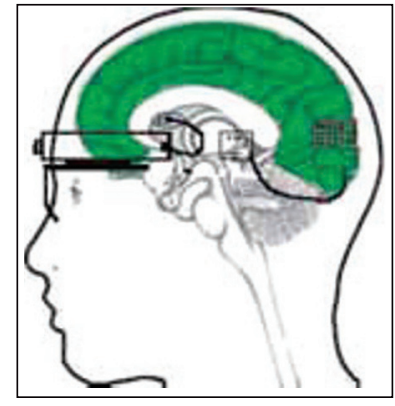

Fig. 4 : Concept of an Intracortical Visual Prosthesis.e perception of an image [3]
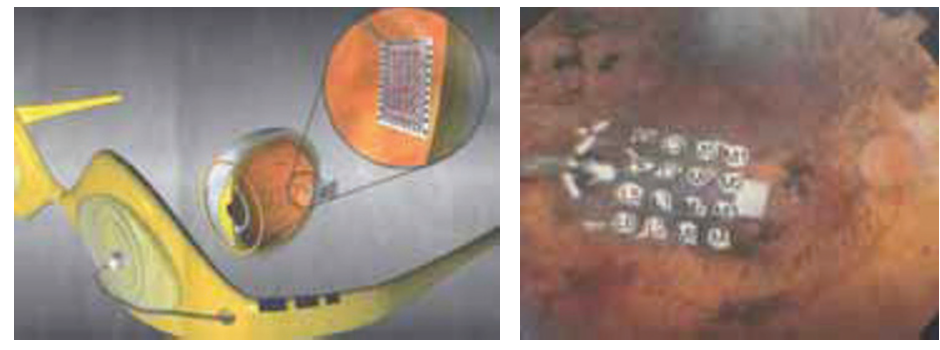

Fig. 6: (a) The concept of an epiretinal prosthesis [19]. (b) Implanted epiretinal prosthesis in a patient of RP [3]
Based on these results a permanent implant trial was started in 2002 at Doheney Retina Institute. In this initial phase, three patients received implants, the 16 electrode model I implant. These patients were able to discern brighter or dimmer precepts with varying levels of current, and the ability to distinguish the direction of motion of objects [11].

While RGCs are located in close proximity to the microelectrode array and require less current, stimulation of these cells would require more image processing and it would be desirable to stimulate the deeper bipolar cells, thereby utilise the intact processing capability of inner retinal cell layers. It has been shown by Greenberg, [3] that by varying the pulse width of the stimulating current the electrodes can be 'tuned' to stimulate selectively retinal bipolar cells rather than RGCs. The RGCs respond to shorter pulse duration (less than $0.5 \mathrm{~ms}$ ) while deeper retinal neural layers responded to longer pulse durations (greater than $0.5 \mathrm{~ms}$ ). This experimental result, if consistently produced in the patient, would enable epiretinal prosthesis to employ the processing from the entire retina as it would initiate the electrical signal propagation at the level of the retinal bipolar cell. The histological retinal changes associated with the mechanical effects of epiretinal implantation are also less severe than with subretinal implantation [12].

The advantages of the epiretinal approach include the following; the vitreous acts as a sink for heat dissipation from the microelectronic device; a minimal number of microelectronics are incorporated into the implantable portion of the device; the wearable portion of electronics allows for easy upgrades without requiring subsequent surgery and the electronics allow the user and the doctor full control over every electrode parameter and digital signal processing involved in imaging objects, allowing the implant to be customised for each patient. The disadvantages of this approach include the requirement of technology to provide prolonged adhesion of the device to the inner retina, and increased current requirement due to greater distance of the target bipolar cells from epiretinal device than with the subretinal device.
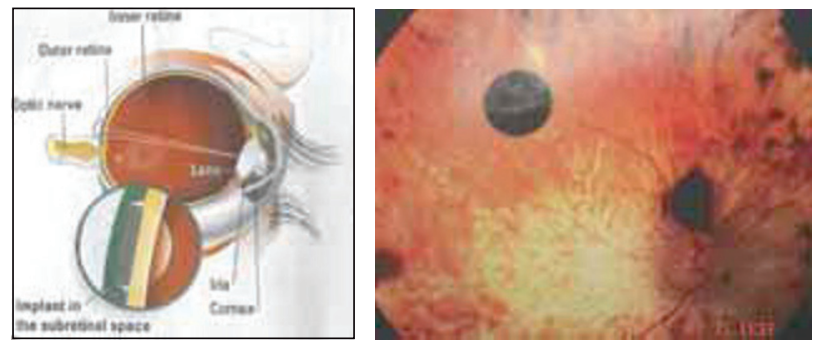

Fig. 7 : (a) The concept of a subretinal implant [19]. (b) A subretinal implant in a patient of RP [19] 


\section{Subretinal Prosthesis}

The subretinal approach to the retinal prosthesis involves implanting a microphotodiode array (MPDA) between the bipolar cell layer and retinal pigment epithelium (Fig.7a,b). This is accomplished surgically either via an intraocular approach through a retinotomy site (ab interno) or a transscleral approach (ab externo).

The subretinal prosthesis, uses microphotodiodes (solar cells) alone as a powering mechanism, which offers an attractive solution to enhance the vision of patients affected by RP and AMD. However, several limitations currently hinder this technology from realising its goal of being a visual prosthesis. The energy efficiency of currently available photodiode arrays is inadequate to generate sufficient electricity for stimulation of bipolar cells [3].

Chow et al [13], have now abandoned the notion that their subretinal implant is a prosthesis, but they claim that the low level of current delivered by the implant has a therapeutic and neuroprotective effect on the otherwise dying retinal photoreceptors.

Zrenner et al [14], have also concluded that a subretinal implant may act as a replacement for lost photoreceptors. Detectable activation of the visual cortex was also achieved in their latest work. They have developed a subretinal prosthesis consisting of an MDPA powered either by a laser or telemetry for the replacement of degenerated photoreceptors in outer retinopathies. They have implanted their subretinal implant in blind patients who have gained a crude form of vision with an ability to recognise forms like a dinner plate and motion [15].

Advantages of the subretinal prosthesis include closer proximity to the next surviving neurons to the visual pathway (i.e. bipolar cell) and therefore less current requirement and the lack of a mechanical means of fixation. The disadvantages include the limited subretinal space to place electronics as well as the close proximity of the retina to the electronics which would increase the risk of thermal injury to the neurons.

\section{Recent Alternative Approaches}

Investigators from Japan have recently collaborated on a relatively new approach to artificial vision that they term as suprachoroidal transretinal stimulation (STS). They have hypothesised that placing a stimulating electrode in the suprachoroidal space or in the fenestrated sclera along with a ground electrode in the vitreous cavity may allow for a less invasive method of achieving functional percepts. The advantages to this approach are that the surgery is less complicated, the electrodes are less invasive to the retina, and can be removed or replaced easily if damaged [16].
Another recent alternative has been the Microfluidic Retinal Prosthesis Implant project developed at the Kresge Eye Institute at Wayne state University (Detroit, USA). This group proposes that this visual prosthesis will mimic normal chemical signaling between the retina and brain [3]. The overall hypothesis is that digital images may be transposed into neurochemical signals through an implanted microfluidic chip that provides continuous meaningful information to the retina and brain.

There is also the Artificial Synapse Chip project at Stanford, USA, that has evolved a design for a flexible retinal interface which aims to improve visual resolution of an electronic retinal prosthesis by addressing cells individually and mimicking the physiological stimulation achieved in synaptic transmission [17]. Both the Kresge and Stanford efforts also have a lot of work to be done before the feasibility of these implants is realised.

\section{Electrotherapeutics}

Two groups Chow et al [13] and the Japanese group [14], are evaluating the benefits of electrotherapeutics. Since the site of implantation of the Chow device is subretinal, it will require viable photoreceptors to be present to function properly. Chow et al have reported improvement in visual function of their patients, which cannot be attributed to a therapeutic effect of low level chronic electric current, but possibly through a yet unknown indirect effect of the implant rather than from a direct activation of neurons. Chow et al reported results of their 10 implanted patients, with 9 to 36 months of follow up. There, were no implant related complications. The improvement in visual function like improved contrast sensitivity and visual fields, have been reported due to an indirect neurotrophic-type rescue effect caused by the mere presence of the implant [12]. The Japanese effort similarly has shown therapeutic effects of electrical stimulation in rabbit eyes [16].

\section{Conclusion}

The exponential advances in the understanding of electronics, physiology and medicine have enabled researchers to change from the earlier Brindley's large, immobile, cortical visual stimulator to the development of implantable electronic retinal prosthesis. Advances in micro technology have allowed for the development of high -density integrated circuit systems that may be implanted either in the epiretinal or subretinal space. Analogous to the cochlear implants for some forms of deafness, these devices are attempting the restoration of useful vision via the remaining spared inner retinal neurons in diseases such as RP and AMD. However, the approach in the visual system is much more complex than the auditory system. In the eye, information processing occurs simultaneously and in parallel within 
millions of neurons that transform the incoming light stimuli into electrical impulses, processing these impulses within the retina before sending the results through axons via the lateral geniculate nucleus to the visual cortex. Thus, the relative complexity of the visual system presents challenges to researchers attempting to develop visual prostheses.

A good beginning has been made, but many areas of research remain for a viable visual prosthesis. Although the presence of viable RGCs and bipolar cells have been documented, the minimum number, type and location of these cells required for a useful prosthesis are currently not known [3]. Biocompatibility of any retinal prosthesis is an issue due to the potential chemical, biophysical, and immunological reactions to the implanted materials and the surgical procedure. Encapsulation with fibrous tissue may occur with any of the implants described, including retinal pigment epithelium reaction to subretinal implants. Such reactions may disrupt contact between the implant and target tissue. Moreover, the potential effects of heat generation and transfer of electrical energy have not been investigated fully by most groups. Finally, many outcome measures remain subjective and only few groups have identified parameters to test subjects [18]. Only through these objective measures can the results be reproducible. It is also to be borne in mind that therapeutic electrical stimulation to treat disease is not same as electrical stimulation to replace lost function which is being attempted by a visual prosthesis. Before concluding it would be justified to add that certain experimental approaches, such as gene and drug therapy, may be a preventive or a therapeutic option.

The number of blind patients is constantly increasing and $10 \%$ of them have no light perception from various causes. The field of visual prostheses has started from a mere dream to early patient trials. It is only with the continued research efforts of the many teams from all over the world that these blind people may have a chance to see again.

\section{Conflicts of Interest}

None identified

\section{References}

1. Weiland J D, Humayun M S. Past, present and future of artificial vision. Artif Organs 2003; 27: 961-2.

2. Hamel C. Retinitis pigmentosa. Orphanet J Rare Dis 2006; 11 : $1-40$.

3. Humayun M S, Lakhanpal R R, Weiland J D. Artificial Vision. In: Stephen J Ryan, Editor. Retina. $4^{\text {th }}$ edition. Philadelphia : Mosby, 2006; 2615 -32.

4. Dobelle WH. Artificial vision for the blind by connecting a television camera to the visual cortex. ASAIO J 2000; 46: 3-9.

5. Troyk PR, Bradley D, Bak M, Cogan S, Erickson R, Hu Z, et al. Intracortical visual prosthesis research - approach and progress.Conf Proc IEEE Eng Med Biol Soc 2005;7:7376-9.

6. Margalit E, Maia M, Weiland JD, Greenberg RJ, Fujii GY, Torres G, et al. Retinal prosthesis for the blind. Surv Ophthalmol 2002; 47: 335-6.

7. Veraart C, Raftopoulos C, Mortimer JT, Delbeke J, Pins D, Michaux G, et al. Visual sensations produced by optic nerve stimulation using an implanted self-sizing spiral cuff electrode. Brain Res 1998;813:181-6.

8. Veraart C, Wanet-Defalque MC, Gérard B, Vanlierde A, Delbeke J. Pattern recognition with the optic nerve visual prosthesis. Artif Organs 2003; 27:996-1004.

9. Kim SY, Sadda S, Humayun MS, de Juan E Jr, Melia BM, Green WR. Morphometric analysis of the macula in eyes with geographic atrophy due to age-related macular degeneration. Retina 2002; 22: 464-70.

10. Kim SY, Sadda S, Pearlman J, Humayun MS, de Juan E Jr, Melia BM,et al. Morphometric analysis of the macula in eyes with disciform age-related macular degeneration. Retina 2002; 22: 471-7.

11. de Balthasar C, Patel S, Roy A, Freda R, Greenwald S, Horsager A, Mahadevappa M, Yanai D, McMahon MJ, Humayun MS, Greenberg RJ, Weiland JD, Fine I. Factors affecting perceptual thresholds in epiretinal prostheses.Invest Ophthalmol Vis Sci 2008; 49: 2303-14.

12. Piyathaisere DV, Margalit E, Chen SJ ,Shyu JS, D'Anna SA, Weiland JD, et al. Heat effects on the retina. Ophthalmic surg lasers Imaging 2003; 34: 114-20.

13. Chow AY, Chow VY, Packo KH, Pollack JS, Peyman GA, Schuchard R. The artificial silicon retina microchip for the treatment of vision loss from retinitis pigmentosa. Arch Ophthalmol 2004; $122: 460-9$.

14. Zrenner E. Restoring neuroretinal function:new potentials. The Emiko Adachi award lecture. Doc Ophthalmol 2007; 115: 56-9.

15. Sachs H G, Bartz-Schmidt K, Gekeler F, Besch D, Brunner U, Wilhelm B, et al. Transchoroidal implantation of active subretinal implants in blind patients: Experience with the new surgical implantation and explantation procedures in the first six patients. Invest Ophthalmol Vis Sci 2007; 48: 4446.

16. Nakauchi K, Fujikado T, Kanda H, Morimoto T, Choi JS, Ikuno $\mathrm{Y}$, et al. Transretinal electrical stimulation by an intrascleral multichannel electrode array in rabbit eyes. Graefes Arch Clin Exp Ophthalmol $2005 ; 243: 169-74$.

17. Peterman MC, Mehenti NZ, Bilbao KV, Lee CJ, Leng T, Noolandi J, et al. The artificial synapse chip:a flexible retinal interface based on directed retinal cell growth and neurotransmitter stimulation. Artif organs 2003; 27: 75-85.

18. Yanai D, Lakhanpal RR, Weiland JD, Mahadevappa M, Van Boemel G, Fujii GY, et al. The value of pre-operative tests in the selection of blind patients for a permanent micro-electric implant. Trans AM Ophthalmol Soc 2003; 101: 223-8.

19. Guttman C. Results remain favourable as retinal prosthesis clinical trial follow up progresses. Eurotimes 2005; 10: 6-7. 